\title{
TENDENCIAS Y AGENDA DE PRIORIDADES DE INVESTIGACIÓN EN UNIDADES ACADÉMICAS DE EDUCACIÓN SUPERIOR DE ENFERMERÍA EN COLOMBIA
}

\author{
TRENDS AND RESEARCH PRIORITIES AGENDA IN HIGHER \\ EDUCATION ACADEMIC UNITS OF NURSING IN COLOMBIA
}

\author{
Consuelo Vélez Álvarez ${ }^{*}$ \\ María Eugenia Pico Merchán ${ }^{* *}$ \\ Claudia Ariza Olarte ${ }^{* * *}$ \\ Fabiola Castellanos ${ }^{* * * *}$ \\ Ángela María Salazar Maya ${ }^{* * * * *}$
}

\begin{abstract}
RESUMEN
Objetivo: Identificar en las unidades académicas miembros de la Asociación Colombiana de Facultades y Escuelas de Enfermería (ACOFAEN), la situación en el componente de investigación, sus fortalezas y necesidades académicas. Material y método: Estudio descriptivo realizado entre 2010 y 2011, universo 30 unidades académicas de enfermería de Colombia, de las que 27 respondieron el instrumento, constituyéndose finalmente en la población del estudio. El instrumento utilizado fue una encuesta diagnóstica autodiligenciada con 123 preguntas, de las cuales 27 correspondían a investigación. La información fue procesada en el programa SPSS versión 11,5; se realizó distribución de frecuencias absolutas y relativas. Resultados: La investigación es transversal en el currículo; el 51\% de las publicaciones se encontraban en revistas nacionales, un $20 \%$ en revistas internacionales. Como líneas temáticas prioritarias se destacan: cuidado por ciclo vital y por áreas temáticas, epidemiología, salud colectiva, epistemología, gestión y gerencia. Como necesidades investigativas: fortalecimiento de competencias por enfoques de investigación y apropiación de metodologías basadas en la evidencia, la Teoría fundada para el abordaje cualitativo. Las prioridades nacionales en investigación fueron: investigación por ciclo vital, por campos de acción, por áreas de desempeño, en problemas prioritarios, investigación en determinantes sociales de la salud, objetivos del milenio, interdisciplinariedad, estrategias de difusión, visibilidad de la producción, nuevos métodos de investigación, más allá de la descripción. Conclusiones: La discusión y el abordaje de la investigación es un elemento que debe integrarse a los currículos; para que la investigación pueda ser un eje articulador en la formación de profesionales de enfermería debe responder a las necesidades de la población y del país.
\end{abstract}

Palabras clave: Investigación en enfermería, tendencias, agenda de prioridades en salud.

\footnotetext{
* Enfermera. Universidad de Caldas, Manizales, Colombia, miembro Comité Permanente de Investigación (COPEI) ACOFAEN. E-mail: consuelo.velez@ucaldas.edu.co

** Enfermera. Universidad de Caldas, Manizales, Colombia, miembro Comité Permanente de Investigación (COPEI) ACOFAEN. E-mail: maria.pico@ucaldas.edu.co

${ }^{* * *}$ Enfermera. Universidad Javeriana, Bogotá, Colombia, miembro Comité Permanente de Investigación (COPEI) - ACOFAEN. E-mail: claariza@javeriana.edu.co

***** Enfermera. Universidad Javeriana, Bogotá, Colombia, miembro Comité Permanente de Investigación (COPEI) - ACOFAEN. E-mail: fabiola.castellano@ javeriana.edu.co

****** Enfermera. Universidad de Antioquia, Medellín, Colombia, miembro Comité Permanente de Investigación (COPEI) ACOFAEN. E-mail: amsalazar@udea.edu.co
} 


\begin{abstract}
Objective: To identify in ACOFAEN academic units members, the situation in research component, the strengths and academic needs. Method: A descriptive study was conducted between 2010 and 2011. 27 academic nursing units from Colombia were included. The instrument used was a diagnostic survey self-served out; 123 questions in total of which 27 corresponded to research. The information was processed using SPSS version 11.5; it was done an absolute and relative frequency distribution. Results: Research is transversal in the curriculum, $51 \%$ of the publications were in national journals, $20 \%$ in international journals. As subject areas include: care for life cycle and by subject, epidemiology, collective health, epistemology, administration and management. As research needs: building skills for research approaches and appropriation of evidence-based methodologies, grounded theory for qualitative approach. The national research priorities were: research for life cycle for action areas, for performance areas in priority problems, research in social determinants of health, millennium development goals, interdisciplinary, dissemination strategies, production visibility, new research methods, beyond description. Conclusion: The discussion and addressing research, is an element that should be integrated into the curriculum, for the research can be a key piece in training nurses, must respond to the needs of the people and the country.
\end{abstract}

Key words: Nursing research, trends, health priority agenda.

Fecha recepción: 15/05/13. Fecha aceptación: 13/08/14.

\section{INTRODUCCIÓN}

Retomando los planteamientos de la Registered Nurses Association of British Columbia (1), la investigación en enfermería es esencial para desarrollar, evaluar y expandir el conocimiento. Constituye una parte vital de la práctica clínica y comunitaria para beneficiar directamente a los usuarios y a través de ella se puede documentar el costo-efectividad de los cuidados basados en evidencia. En este mismo sentido, Castrillón plantea cómo es necesario hacer uso de los recursos en forma eficiente sin afectar la calidad y teniendo una definición clara de las prioridades en la producción de conocimiento de enfermería, de tal forma que respondan tanto a la necesidad de fundamentar la ciencia de la profesión como a las necesidades en salud (2).

Según el Nursing Education Advisory Council de la Organización "National League for Nursing" (3), para lograr excelencia en investigación en enfermería, tanto académicos como estudiantes deben contribuir al desarrollo de la ciencia a través de la crítica, utilización, diseminación y conducción de investigaciones. Docentes y alumnos deben discutir sobre investigaciones realizadas y su aplicación en la profesión y su desarrollo.

En la investigación en enfermería otro aspecto de gran relevancia es el abordaje metodológico dado al proceso y en este sentido, tal como lo plantean algunos autores, la adopción de métodos y enfoques teóricos provenientes de otras disciplinas ha requerido la apropiación de sus contenidos y fundamentos, lo que ha provocado, por una parte, una expansión de posibilidades para la construcción de conocimientos y perfeccionamiento de los profesionales desde el punto de vista científico y cultural y, por otra, implica que los/las investigadores/as se adscriban acríticamente al paradigma cualitativo o cuantitativo (4).

De acuerdo con Murrain (5), actualmente en el plano internacional han surgido tres temáticas generales que podrían constituirse como prioritarias para la investigación en enfermería: a) los principios y leyes que orientan los procesos relacionados con la vida, 
el bienestar y el funcionamiento adecuado de los seres humanos tanto en situación de enfermedad como de salud; b) las pautas de conductas y comportamiento del ser humano y su interacción con el entorno o medio ambiente, cuando la persona se enfrenta a situaciones críticas de la vida; y c) los procesos por los cuales se impactan los factores y campos favorecedores del estado de la salud.

En este mismo sentido, es importante retomar lo planteado por el Consejo Internacional de Enfermería (CIE) (6), que en el 2007 ratificó la Declaración de Posición frente a la investigación en enfermería, en la que se enfatiza que la práctica basada en la investigación se constituye en un distintivo de la enfermería profesional, tan necesario para generar nuevos desarrollos teóricos, permitir el avance de la ciencia de enfermería, evaluar la práctica y los servicios actuales como problemática social que aportan evidencia científica en la formación, la práctica, la gestión del cuidado.

El CIE identificó, dentro de las prioridades para la investigación de enfermería, las líneas de acción que resaltan la calidad de la atención y su eficacia en términos de costos, los cuidados basados en la comunidad, la fuerza de trabajo de la enfermería en cuanto a sus condiciones laborales y las reformas relativas a la atención de la salud (6).

El estudio de Mendoza et al. (7) realizado el 2009, relacionado con la visibilidad de la investigación de Enfermería en América Latina, describió el universo de las revistas de enfermería del área -119-, la mayoría documentadas en el Directorio de acceso libre de Publicaciones de Enfermería en América Latina de la Organización Panamericana de la Salud (60\%); además de 13.208 documentos científicos extraídos de las bases de datos de internet: Web of Science (WOS), Medline, LILACS, Periodica, Cuiden, y SciELO. Así durante el periodo del estudio, 1959-2005, el país que estuvo en la delantera en producción científica fue Brasil (31,9\%), a continuación los países del cono suramericano (25\%), de los cuales se destaca Argentina con un 16\% y Colombia con un $12,6 \%$ como representante de la región andina; España, Estados Unidos e Inglaterra son los países que más han contribuido a la difusión de la producción en enfermería.

Del mismo modo autores $(8,9)$, resaltan lo expuesto por Cianelli et al. en el 2009 (10) frente a las dificultades que en América Latina tienen los profesionales de enfermería para la investigación, dificultades que van desde la carencia de financiación hasta los problemas para acceder a publicar en las revistas de alto nivel; además los problemas se agravan por la preparación inadecuada y la falta efectiva de tiempo, agregado a la sobrecarga de trabajo y a la percepción de dominio de esta área como compleja $(11,12)$. De ahí que los autores propongan la necesidad de consolidar alianzas de investigación interinstitucionales y centros de investigación específicos para tan importante labor, con el fin de plantearse objetivos globales en materia de salud para incrementar la producción de conocimiento en áreas que apoyan tanto la disciplina de enfermería como la investigación y de acuerdo a las necesidades particulares de la sociedad (8).

Alarcón y Astudillo (13) encontraron, en una investigación de corte transversal, que entre las temáticas trabajadas en las tres revistas revisadas aparecen las relativas a la percepción, conocimiento y creencias de pacientes y sus familias (14,6\%), el análisis de prevalencia e incidencia de patologías y diferentes problemáticas $(13,2 \%)$ y las relacionadas con la evaluación de competencias profesionales en enfermería (12,6\%).

Para el caso de Colombia, el estudio realizado por Arango y Ayala (9) en el periodo 1969-2009 evidenció un incremento en las publicaciones académicas a partir del año 1976, gracias a la Revista de la Asociación Nacional de Enfermeras de Colombia (ANEC), alcanzando un incremento a partir del año 2008 con el logro de publicaciones en revistas internacionales y la aparición de revistas nacionales que fueron indexadas en las máxi- 
mas categorías de Colciencias, la entidad que en Colombia las clasifica según su calidad en la producción científica.

Con el de desarrollo de este trabajo se pretendió responder a la pregunta sobre la situación de la investigación en las unidades académicas de la educación superior de enfermería en Colombia y plantear algunas tendencias y agenda de prioridades. De este modo, el objetivo fue identificar, en las unidades académicas miembros de la Asociación Colombiana de Facultades y Escuelas de Enfermería (ACOFAEN), la situación de la investigación, sus fortalezas y necesidades académicas para llegar a determinar tendencias, sin perder de vista que quedan muchos retos y uno de los más importantes es que la investigación se haga evidente en las diferentes agendas de los programas del país.

\section{MATERIAL Y MÉTODO}

Estudio descriptivo cuyo universo estuvo conformado por 30 unidades académicas de enfermería del país miembros de ACOFAEN, de las que 27 respondieron y diligenciaron el instrumento, constituyéndose finalmente en la población del estudio; la información fue recopilada entre 2010 y 2011. El instrumento utilizado fue una encuesta diagnóstica autodiligenciada en cada unidad por la respectiva directora y su equipo de trabajo, conformada por los siguientes ítemes: aspectos generales, desarrollo disciplinar y curricular, desarrollo investigativo, humanización ética y bioética, movilidad académica, docencia, servicio, educación continuada, egresados y sistema de comunicaciones; 123 preguntas en total, de las cuales correspondían al componente de investigación 27, estas últimas se resumen en las siguientes variables: características de la investigación formativa; grupos, líneas y enfoques de investigación; clasificación de los grupos en Colciencias; apoyos institucionales para investigación en enfermería; vigencia de los proyectos de investigación; número y calidad de publicaciones científicas, redes de investigación en las que participan; inclusión de la Enfermería Basada en la Evidencia y su relación con el currículo y estrategias para su implementación; revistas de difusión del conocimiento de enfermería; otras publicaciones; fundamentación teórica de la investigación en enfermería; necesidades de capacitación y asesoría en investigación.

La encuesta fue enviada por correo electrónico a las unidades académicas para su diligenciamiento, posteriormente fue procesada en el programa SPSS versión 11,5; sobre los resultados se realizó un análisis descriptivo realizando distribución de frecuencias absolutas y relativas. El estudio se acogió a los lineamientos de la Resolución 8430 de 1993 del Ministerio de Salud (Colombia), considerada investigación sin riesgo, en razón a que no se realizó ninguna intervención sobre sujetos, puesto que la encuesta fue efectuada por cada unidad académica de los programas de enfermería.

\section{RESULTADOS}

Los resultados del trabajo se consolidaron en las siguientes categorías de análisis que presentan un panorama general de la investigación en enfermería en las unidades académicas de educación superior en Colombia.

\section{Investigación en el currículo de forma- ción}

Frente a la modalidad de investigación en los programas de enfermería en el pregrado las unidades académicas informaron que en 17 (63\%) de ellas se trabaja de manera transversal en el currículo, 5 (19\%) respondieron que se da por la modalidad de curso de investigación, $3(11 \%)$ por proceso y trabajo de investigación, 2 (7\%) tienen la modalidad de proyecto de grado. 
Al indagar en las unidades académicas sobre la inclusión en el proyecto curricular del tema de enfermería basada en la evidencia $(\mathrm{EBE})$, se encontró que a nivel de pregrado está incluido en $16(59,2 \%)$ de las 27 y en posgrado en $5(18,5 \%)$ de las 27.

\section{Producción en investigación}

Al momento de la encuesta, el mayor porcentaje de los grupos de investigación en enfermería clasificados por el Departamento Administrativo de Ciencia, Tecnología e Innovación (Colciencias), se encontraba en las categorías C y D (55,9\%), la máxima categoría en enfermería para los grupos de investigación corresponde a "A" con 6,9\%; nótese que el 18,6\% aparece como "no escalafonados" a pesar de encontrarse registrados (Tabla 1). Es importante comentar que en la actualidad está en proceso de cambio el sistema de escalafonamiento de los grupos de investigación por parte de Colciencias.

En la Tabla 2 se presentan el total de publicaciones por las diferentes unidades académicas en los últimos 2 años $(2010,2011)$,

Tabla 1. Clasificación de los grupos de investigación de los programas de enfermería según Colciencias. Colombia 2011.

\begin{tabular}{lrr}
\hline Clasificación según Colciencias & n & \% \\
\hline Grupo A & 4 & 6,9 \\
Grupo B & 11 & 18,6 \\
Grupo C & 12 & 20,3 \\
Grupo D & 21 & 35,6 \\
Registrado & 11 & 18,6 \\
\hline Total & 59 & 100 \\
\hline
\end{tabular}

Tabla 2. Publicaciones de artículos en enfermería en los últimos 2 años. Colombia 2011.

\begin{tabular}{lrc}
\hline Publicaciones de artículos en enfermería en los últimos 2 años & n & \% \\
\hline Revistas indexadas nacionales & 182 & 51 \\
Revistas homologadas internacionales & 72 & 20 \\
Texto o libros de investigación & 54 & 15 \\
Redes académicas virtuales & 49 & 14 \\
\hline Total & 357 & 100 \\
\hline
\end{tabular}

Tabla 3. Clasificación de las revistas adscritas a los programas de enfermería según Colciencias. Colombia 2011.

\begin{tabular}{lrc}
\hline Clasificación de las revistas & n & \% \\
\hline A2 & 3 & 15,8 \\
B & 2 & 10,5 \\
C & 3 & 15,8 \\
No Clasificada & 11 & 57,9 \\
\hline Total & 19 & 100 \\
\hline
\end{tabular}


resaltando que el $51 \%$ se encontraban en revistas indexadas nacionales, y un $20 \%$ en revistas homologadas por Colciencias de carácter internacional. 19 unidades académicas $(70 \%)$ cuentan con revistas para la difusión del conocimiento de la investigación en enfermería; de éstas, el 15,8\% pertenece a categoría A2 y el 57,9\% no se encuentra clasificada (Tabla 3); al momento de realizar esta encuesta no estaba registrada Aquichán, que en la actualidad está en A1.

\section{Líneas temáticas y enfoques de investiga- ción en pregrado y posgrado}

Como líneas temáticas que han sido abordadas de manera prioritaria en la investigación en enfermería en Colombia se encuentran: Cuidado por ciclo vital y por áreas temáticas, epidemiología-salud colectiva, epistemología, gestión y gerencia.

\section{Necesidades de asesoría y capacitación}

Al realizar el diagnóstico de las necesidades de asesoría y capacitación se encontró que las unidades académicas identifican la actualización en el manejo del software, asesoría en procesos de manejo de tecnología, bases de datos, capacitación en la elaboración de presupuestos, capacitación sobre la investigación cuantitativa y cualitativa (fortalecimiento de competencias en los diferentes enfoques y metodologías de investigación), conceptualización de metodologías investigativas en enfermería para el desarrollo de la EBE, desarrollo y fortalecimiento de competencias para escribir y analizar artículos científicos.

\section{Estrategias metodológicas}

Las unidades académicas proponen como estrategias metodológicas para el abordaje de investigación en enfermería en Colombia el análisis de publicaciones de investigación, capacitación en la investigación cualitativa basada en la EBE, cursos y talleres con exper- tos o a través de la web, educación continua para docentes de los programas de enfermería, elaboración conjunta de guías y protocolos con instituciones prestadoras de servicios de salud, elaboración de guías de laboratorio, encuentros y grupos de estudio, intercambio de docentes, pasantías en otras universidades, trabajos conjuntos con otras universidades, realizar convenios interinstitucionales, seminarios y suscripción a revistas indexadas.

\section{Política institucional en investigación}

Con relación a la política institucional de apoyo a la difusión de conocimiento producido por enfermería, 22 (81,5\%) unidades académicas reportaron que sí existe y 5 $(18,5 \%)$ en las que no existe, las formas bajo las cuales se expresa la política se encuentra dada por sello editorial, revista, oficina de investigaciones y política de investigación.

\section{Prioridades nacionales}

Como prioridades nacionales en investigación a partir de los resultados de este trabajo fueron categorizadas las siguientes: investigación por ciclo vital, investigación por campos de acción, investigación por áreas de desempeño, investigación por problemas prioritarios, agendas regionales, investigación en determinantes sociales de la salud, objetivos del milenio, investigación en equipos interdisciplinarios, unificación de estrategias de difusión de la investigación, trabajo en red y visibilidad de la producción, retomar nuevos métodos de investigación, más allá de la descripción, registro en bases de datos internacionales, definir un plan de capacitación en investigación para enfermería.

\section{DISCUSIÓN Y CONCLUSIÓN}

La investigación en enfermería se ha convertido en un reto no sólo para la profesión, sino 
para las universidades, y en este sentido, tal como se encontró en Colombia, existen una serie de políticas institucionales como oficina de investigaciones, política de investigación propiamente dicha, sellos editoriales entre otros, que buscan dar legitimidad al proceso. Esta prioridad está siendo asumida además en el contexto internacional, tal como lo presenta Zárate (14), resaltando la necesidad que las políticas consoliden la investigación y direccionen acciones estratégicas para que sea asumida como función sustantiva institucional y de programas.

En Colombia las diferentes unidades académicas tienen la modalidad de investigación de manera transversal en el currículo, y un bajo porcentaje trabaja la investigación mediante curso de investigación. Lo anterior hace evidente que se requiere incorporar estrategias para desarrollar el espíritu científico desde los primeros semestres, con el fin de ir construyendo el camino para fortalecer la formación en niveles más avanzados, buscando que el estudiante adquiera los conocimientos y habilidades necesarias para la investigación (15).

En la categoría líneas temáticas y enfoques de investigación a nivel de pregrado y posgrado en Colombia, se identificaron como prioritarias las relativas a cuidado por ciclo vital, epidemiología, salud colectiva y epistemología, datos que concuerdan con lo encontrado por Ailinger et al., quienes reportan que de un total de 263 trabajos presentados en Coloquios Panamericanos de Investigación en Enfermería la salud pública fue la temática principalmente abordada, seguida de recursos humanos y en menor porcentaje el entorno clínico (16). En contraste, lo reportado por Heitkemper (17) hace relación a la necesidad de un mayor desarrollo de la investigación en enfermería en los Estados Unidos y países asiáticos en las áreas de autogestión, genética, geriatría, la promoción de la salud según ciclo vital, tecnología y salud mental; al igual que Comet et al. (18), quienes hallaron que los cuidados basados en la evidencia, la implantación y evaluación de resultados en la práctica clínica y la medición de la calidad de cuidados enfermeros son prioridades de investigación importantes en España, entre otras.

Recientemente de acuerdo a lo reportado por Varela et al. (19), se definen como focos temáticos para el área asistencial los relativos a profundizar en el conocimiento del ser humano en cuanto a su singularidad, bienestar y confort y la identificación de necesidades reales de los pacientes, dando respuesta a sus expectativas y además a la validación de instrumentos de la práctica clínica. De igual manera otras investigaciones plantean que es necesario reflexionar sobre los retos y contribuciones que la investigación en resultados en enfermería puede aportar en los próximos años (20).

Una de las estrategias metodológicas propuestas para la labor en investigación en enfermería por las unidades académicas en Colombia es el trabajo conjunto con otras universidades, el cual es posible lograr cuando se impulsa el trabajo en redes articuladas a las tecnologías de la información y la comunicación, tal como lo describen Quintana y Paravic en su texto de internacionalización de la educación en enfermería y sus desafíos (21).

Retomando los planteamientos internacionales, donde las tendencias orientan hacia el impulso del trabajo colaborativo e interdisciplinario y la creación de centros internacionales de investigación en enfermería y además la relevancia de la tutoría a futuros científicos (17), los hallazgos de este trabajo resultan coherentes con este planteamiento ya que las unidades académicas las informaron como prioridades y necesidades nacionales.

La difusión de los resultados de la investigación en Enfermería en Colombia en los últimos años se ha venido dando en mayor porcentaje en revistas nacionales indexadas, las unidades académicas han realizado un importante esfuerzo en contar con revistas que les permitan publicar sus trabajos, es así 
que se convierte en un reto para la investigación en enfermería en Colombia contar con alianzas estratégicas para la internacionalización de la producción investigativa, no sólo en lo relacionado con publicaciones científicas sino en el fortalecimiento de sus grupos de investigación, este mismo planteamiento coincide con Quintana y Paravic, quienes resaltan la importancia de impulsar la internacionalización de la educación y la investigación en enfermería, y finalizar los procesos de investigación con publicaciones científicas (21).

Como estrategia se hace necesario que la práctica de la enfermería se apoye en la transferencia de conocimiento, basado en investigación que genere conocimientos válidos y basados en la evidencia (22). Complementario a lo anterior, se propone el método meta-síntesis como otra metodología que fortalece el proceso investigativo a nivel cualitativo basado en un modelo crítico reflexivo de revisión, síntesis y difusión de evidencias $(23,24)$, además de la teoría fundamentada en datos (TDF), considerada como un referencial interpretativo que retoma de las experiencias vivenciadas por los actores sociales, en este caso pacientes y familia, aspectos significativos que permitan construir bases teóricas $(25,26)$.

Las prioridades nacionales en investigación en enfermería en Colombia encontradas en este trabajo concuerdan con los resultados de estudios que resaltan: cómo los temas de salud pública y atención primaria de salud deben ser retomados en el contexto internacional de la investigación en la profesión (27-29).

Para futuras investigaciones el reto sería la posibilidad de realizar un estudio con una muestra probabilística de las enfermeras que realizan investigación en el país y obtener la visión del investigador propiamente dicha.

A manera de conclusión se resaltan aspectos como: La necesidad de retomar las agendas de investigación regionales; integración de la discusión y el abordaje de la investiga- ción a los currículos; responder a las necesidades de la población y del país para que la investigación pueda ser un eje articulador en la formación de profesionales de enfermería y la formación por competencias en investigación debe considerarse una prioridad en los currículos de pre y posgrado.

Agradecimientos: A la Junta Directiva de la Asociación Colombiana de Facultades de Enfermería (ACOFAEN) por facilitar el acceso a la base de datos referente al objeto de este trabajo.

\section{REFERENCIAS}

1. Registered Nurses' Association of British Columbia. Nursing and Research. Nurs BC. 2003; 35(1): 29-30.

2. Castrillón MC. Trends and priorities in nursing research. Rev Lat Am Enfermagem. 2004; 12(4): 583-588.

3. National League for Nursing. Hallmarks of excellence in nursing education. Nursing Education Perspective. 2004; 25(2): 98-101.

4. Salazar A, Paravic T. Los métodos cualitativo y cuantitativo en la enseñanza de la investigación en enfermería. Rev Cubana Enferm [Internet]. 2009 ene-jun [citado14 may 2013]; 25(1-2). [8 p.] Disponible en: http://scielo.sld.cu/scielo.php?pi$\mathrm{d}=$ S0864-03192009000100008\&script $=\mathrm{s}-$ ci_arttext

5. Murrain E. Tendencias de la investigación en enfermería. Repert. med. cir. 2009; 18(2): 90-96.

6. Consejo Internacional de Enfermería. Declaración de Posición. Investigación de enfermería [Internet]. Ginebra: Consejo Internacional de Enfermería; 2007 [citado 12 marzo 2013]. 2 p. Disponible en: http://www.icn.ch/es/publications/position-statements/ 
7. Mendoza S, Paravic T, Muñoz AM, Barriga OA, Jiménez E. Visibility of Latin American nursing research (1959-2005). J Nurs Scholarsh. 2009; 41(1): 54-63.

8. Ayala R, Sanhueza O. [Chili]. Rech Soins Infirm. 2010; 100: 28-31.French.

9. Arango G, Ayala R. [Colombie]. Rech Soins Infirm. 2010; 100: 32-35. French.

10. Cianelli R, Urrutia M, Ferrer L, Poupin L, Masalán P, Villegas N, et al. Desarrollo de la investigación en enfermería: estudio diagnóstico. Invest Educ Enferm. 2009; 27(1): 112-117.

11. Orellana A, Sanhueza O. Competencia en investigación en enfermería. Cienc. enferm. 2011; XVII(2): 9-17.

12. Moreno T, Fuentelsaz C, González E, Gil A. Barreras para la utilización de la investigación. Estudio descriptivo en profesionales de enfermería de la práctica clínica y en investigadores activos. Enferm Clin. 2010; 20(3): 53-164.

13. Alarcón AM, Astudillo P. La investigación en enfermería en revistas latinoamericanas. Cienc. enferm. 2007; XIII(2): 25-31.

14. Zárate R. Situación de la Investigación en enfermería en Méjico. Cultura de los cuidados. 2011; 15(31): 9-15.

15. ALADEFE, Universidad de Antioquia. VII Conferencia Iberoamericana de Educación en Enfermería: Necesidades, Tendencias y Desafíos. Formación de investigadores de enfermería [Internet]. Biblioteca Lascasas. Medellín; 2006 Oct [citado 14 may 2013]. 29 p. Disponible en http:// www.index-f.com/lascasas/documentos/ lc0156.pdf

16. Ailinger R, Nájera RM, Castrillón MC, Manfredi M. Nursing research in Latin America: 1988-1998. Rev Lat Am Enfermagem. 2005; 13(6): 925-928.

17. Heitkemper M. The past and future of Nursing Research. Asian Nurs Res (Korean Soc Nurs Sci). 2007; 1(1): 4-10.

18. Comet P, Escobar G, González T, De Ormijana A, Rich M, Vidal C, et al. Establecimiento de prioridades de investigación en enfermería en España: estudio Delphi. Enferm Clin 2010; 20(2): 88-96.

19. Varela MD, Sanjurjo ML, Blanco FJ. La Investigación en enfermería. Rol de la enfermería. ENFURO. 2012; 121: 19-21.

20. Morales JM, Morilla JC, Torres L, Porcel AM, Canca JC. Investigación en resultados y clasificación de resultados de Enfermería. Claves para la investigación. Metas de Enfermería. 2012; 15(10): 20-24.

21. Quintana MO, Paravic T. Internacionalización de la Educación en Enfermería y sus desafíos. Enfermería global. 2011; 10(4): 200-209.

22. Wallin L. Knowledge translation and implementation research in nursing. Int J Nurs Stud. 2009; 46(4): 576-587.

23. Romero MN. Bases conceptuales de la enfermería basada en la evidencia. Anales de la IV Jornada Internacional de Investigación en Ciencias de la Enfermería; 2010 Jun 24-26; Chiclayo, Perú. Chiclayo (PE): Universidad Católica Santo Toribio de Mogrovejo; 2010.

24. Vásquez ML. Cuidar e investigar: desafíos metodológicos en enfermería. Texto contexto-enferm. 2011; 20(1): 175-183.

25. Soares de Lima S, Leite J, Lorenzini A, Giacomelli A, Andrade M, Real Lima V. La teoría fundamentada en Datos: un camino a la investigación en enfermería. Index Enferm. 2010; 19(1): 55-59.

26. Vivar C, Arantzamendi M, López-Dicastillo O, Gordo C. La teoría fundamentada como metodología de investigación cualitativa en enfermería. Index Enferm. 2010; 19(4): 283-288.

27. Organización Panamericana de la Salud. Agenda de salud para las Américas, 2008-2017 [Internet]. Panamá: Organización Panamericana de la Salud; 2007 Jun [citado 14 may 2013]. 23 p. Disponible en: http://www1.paho.org/hq/dmdocuments/2009/Agenda_Salud_para_las_ Americas_2008-2017.pdf

28. Canadian Nurses Association. Determinants of health [Internet]. Otawa: $\mathrm{Ca}-$ 
nadian Nurse Association; 2009 Nov [citado 14 may 2013]. 5 p. Disponible en: http://cna-aiic.ca/ /media/cna/files/en/ ps124_social_determinants_of_health_e. pdf?la=en
29. Díaz L, Bueno L, Mejía M, López L. Investigación de enfermería: una caracterización en dos publicaciones latinoamericanas. av.enferm. 2011; 29(2): 285-293. 